\title{
Carrier Device
}

National Cancer Institute

\section{Source}

National Cancer Institute. Carrier Device. NCI Thesaurus. Code C49863.

A device designed to facilitate the support, movement, or transport of another device or object. 